\title{
Análise dos óbitos decorrentes de intoxicação ocorridos no Brasil de 2010 a 2015 com base no Sistema de Informação sobre Mortalidade (SIM)
}

\author{
Analysis of deaths by intoxication that occurred in Brazil from 2010 \\ to 2015 based on the Mortality Information System (SIM)
}

Rosany Bochner (https://orcid.org/0000-0002-8797-8874) ${ }^{1}$

Marina Moreira Freire (https://orcid.org/0000-0002-5039-2407) ${ }^{1}$

${ }^{1}$ Instituto de Comunicação

e Informação Científica

e Tecnológica em

Saúde, Fiocruz. Avenida

Brasil 4365/Pavilhão

Haity Moussatché/206,

Manguinhos. 21045-900

Rio de Janeiro RJ Brasil

robochner@gmail.com

\begin{abstract}
Many studies analyze the epidemiological profile of deaths caused by a single toxic agent. However, broader mortality analyses can be obtained by evaluating multiple agents over the same period of time. For this purpose, a retrospective descriptive study was carried out of the deaths by intoxication registered in the Mortality Information System that occurred in Brazil from 2010 to 2015. Deaths were selected according to ICD-10 codes related to intoxication. There were 18,247 deaths and an increase of $3 \%$ of rates of mortality by intoxication during the period. The agents that caused the most deaths were pesticides (24\%) followed by medication (23\%) and street drugs (22\%). With the exception of medication, where the female participation was 52\%, there was a higher concentration of males for all agents and in most of the age groups. Only in the case of medication and pesticides was suicide the main circumstance of deaths. The results presented made it possible to define a mortality profile for each of the major toxic agents studied.
\end{abstract}

Key words Mortality, Intoxication, Medication, Pesticides, Street drugs
Resumo Muitos estudos analisam o perfil epidemiológico dos óbitos causados por um único agente tóxico. No entanto, análises mais amplas da mortalidade podem ser obtidas avaliando múltiplos agentes em um mesmo período de tempo. Com esse objetivo, foi realizado um estudo descritivo retrospectivo dos óbitos decorrentes de intoxicações ocorridos de 2010 a 2015 no Brasil, registrados pelo SIM. Os óbitos foram selecionados de acordo com os códigos da CID-10 relacionados a intoxicações. Foram registrados pelo SIM, no período do estudo, 18.247 óbitos decorrentes de intoxicação, resultando em crescimento de $3 \%$ no coeficiente de mortalidade. Os agentes que mais causaram óbitos foram os agrotóxicos (24\%) seguidos dos medicamentos (23\%) e das drogas de abuso (22\%). Com exceção dos medicamentos, em que a participação do sexo feminino foi de $52 \%$, verificou-se maior concentração do sexo masculino para todos os agentes e na maioria das faixas etárias. Somente para os medicamentos e agrotóxicos, o suicídio foi a principal circunstância dos óbitos. Os resultados apresentados foram capazes de delinear um perfil de mortalidade para cada um dos principais agentes tóxicos estudados.

Palavras-chave Mortalidade, Intoxicação, Medicamentos, Agrotóxicos, Drogas de abuso 


\section{Introdução}

No Brasil, as causas externas ocupam o segundo lugar nas estatísticas de mortalidade ${ }^{1}$. Dentre os óbitos por causas externas, podemos dar destaque aqueles causados por intoxicação, dado a severidade de suas consequências e do crescimento de sua ocorrência. A Organização Mundial de Saúde considera as intoxicações como um importante agravo de Saúde Pública, que deve ser investigado com consequente geração de informações que fomentem a capacidade dos países para lidar com essa questão².

Dado que as intoxicações constituem um problema de saúde pública em todo o mundo, deve-se ter em mente, para uma correta determinação da epidemiologia de seus óbitos, que este agravo tem peculiaridades geográficas, sociais, econômicas e culturais, que determinam perfis diferentes entre os países e até entre as regiões geográficas de um mesmo país ${ }^{3}$. Desta forma, é necessária a análise quantitativa e qualitativa deste agravo à saúde. Segundo Laurenti et al. ., "as estatísticas de mortalidade constituem os melhores indicadores para identificar os problemas de saúde da população".

A análise da mortalidade permite a avaliação da situação de saúde no tempo utilizando variáveis como sexo, faixa etária e causas; a identificação de grupos e áreas de risco para diferentes agravos, incluindo as intoxicações; e a construção de indicadores de saúde tais como a mortalidade prematura, expressa em Anos Potenciais de Vida Perdido (APVP) $)^{4,5}$.

O APVP traz informações tanto da extensão da mortalidade na população, descrita por meio das taxas de mortalidade e da mortalidade proporcional, quanto quantificam o impacto resultante desta perda para a sociedade, qualificando estas mortes. A morte precipitada de uma pessoa traz consequências não apenas para ela e sua família, mas impede toda a sociedade do contato com seu potencial econômico, cultural e intelectual ${ }^{6}$.

$\mathrm{Na}$ análise dos indicadores de mortalidade, $\mathrm{o}$ Sistema de Informação Sobre Mortalidade (SIM) assume um papel fundamental na medida que possui variáveis que permitem, a partir da causa mortis atestada pelo médico, construir indicadores e processar análises epidemiológicas que contribuam para a eficiência da gestão em saúde.

Muitos estudos analisam o perfil epidemiológico dos óbitos causados por um único agente tóxico, como Mota et al. ${ }^{7}$, que traçaram o perfil da mortalidade por intoxicação com medicamentos no Brasil para o período de 1996 a 2005 e Santana et al. ${ }^{8}$, que analisaram a mortalidade por intoxicação ocupacional relacionada a agrotóxicos no Brasil no período de 2000 a 2009. No entanto, há carência de trabalhos que contemplem mais de um agente em um mesmo período, apontando a necessidade de estudos mais amplos, capazes de realizar comparações e apresentar um perfil menos segmentado.

Intoxicações causadas por múltiplos agentes, ou pelo uso de mais de um agente tóxico em situações de tentativa de suicídio, por exemplo, são bastante comuns. Além disso, distintas substâncias químicas classificadas em grupos químicos diferentes, apresentam o mesmo perfil social de usuário e até mesmo efeitos bastante similares. Desta forma, a análise dos óbitos por intoxicação levando em consideração a gama de substâncias mais prevalentes se mostra bastante necessária, embora complexa e laboriosa.

A análise dos óbitos no Brasil, decorrentes de intoxicação considerando múltiplos agentes, revela-se de grande importância para a Saúde Pública, pois evidencia informações novas, que podem subsidiar intervenções específicas em saúde, gerando ações e políticas melhor direcionadas.

Diante disso, o objetivo deste estudo é traçar o perfil epidemiológico dos óbitos decorrentes de intoxicação ocorridos de 2010 a 2015 no Brasil, registrados pelo SIM.

\section{Metodologia}

Foram coletados no sítio eletrônico do Departamento de Informática do Ministério da Saúde (DATASUS $)^{9}$ os bancos de dados contendo as declarações de óbito (DO) para os anos de 2010 a 2015, disponibilizados por UF.

Neste estudo, foi considerado como caso de "óbito decorrente de intoxicação" todo registro de paciente notificado no SIM que apresentou como causa básica de óbito códigos da $10^{\mathrm{a}} \mathrm{Re}$ visão da Classificação Internacional de Doenças $(\mathrm{CID}-10)^{10}$ associados à intoxicação.

O Quadro 1 apresenta os códigos utilizados da CID-10 relacionados à intoxicação, considerados neste estudo.

Com o banco já selecionado pela variável de interesse "causa básica" de forma a termos somente os óbitos decorrentes de intoxicação, foram realizadas análises com base nas variáveis agente tóxico, UF, região geográfica, sexo, idade, circunstância, escolaridade.

A variável agente tóxico foi criada a partir dos códigos presentes na variável causa básica, des- 
critos no Quadro 1, da seguinte forma: medicamentos - F13.0, F15.0, P93, X40, X41, X43, X44, X60, X61, X63, X64, Y10, Y11, Y13, Y14, agrotóxicos - X48, X68, X87, Y18, drogas de abuso - F10.0, F11.0, F12.0, F14.0, F16.0, F17.0, F19.0, X42, X62, $\mathrm{Y} 12$, produtos químicos industriais - F18.0, X46, X47, X66, X67, X86, X88, X89, X90, Y16, Y17, alimentos - A02, A05, plantas - X28, X29, medica- mentos + drogas de abuso - X85, drogas de abuso + produtos químicos industriais - X45, X65, Y15, miscelânea (produtos químicos industriais + domissanitários + plantas + metais + alimentos) X49, X69, Y19. A combinação de agentes tóxicos se fez necessária, uma vez que alguns códigos da CID-10 não são suficientemente específicos para caracterizar um único agente.

Quadro 1. Códigos da CID-10 relacionados a intoxicação.

\begin{tabular}{|c|c|}
\hline Código & Capítulo/ Descrição \\
\hline \multicolumn{2}{|r|}{ Capítulo I: Algumas Doenças Infecciosas e Parasitárias } \\
\hline A02.0 & Enterite por salmonela \\
\hline A02.1 & Septicemia por salmonela \\
\hline A02.2 & Infecções localizadas por salmonela \\
\hline A02.8 & Outras infecções especificadas por salmonela \\
\hline A02.9 & Infecção não especificada por salmonela \\
\hline A05.0 & Intoxicação alimentar estafilocócica \\
\hline A05.1 & Botulismo \\
\hline A05.2 & Intoxicação alimentar devida a Clostridium perfringens [Clostridium welchi] \\
\hline A05.3 & Intoxicação alimentar devida a Vibrio parahemolyticus \\
\hline A05.4 & Intoxicação devida a Bacillus cereus \\
\hline A05.8 & Outras intoxicações alimentares bacterianas especificadas \\
\hline A05.9 & Intoxicação alimentar bacteriana não especificada \\
\hline \multicolumn{2}{|r|}{ Capítulo V: Transtornos Mentais e Comportamentais devidos ao uso de } \\
\hline F10.0 & Álcool - intoxicação aguda \\
\hline F11.0 & Opiáceos - intoxicação aguda \\
\hline F12.0 & Canabinóides - intoxicação aguda \\
\hline F13.0 & Sedativos e hipnóticos - intoxicação aguda \\
\hline F14.0 & Cocaína - intoxicação aguda \\
\hline F15.0 & Outros estimulantes, inclusive a cafeína - intoxicação aguda \\
\hline F16.0 & Alucinógenos - intoxicação aguda \\
\hline F17.0 & Uso de fumo - intoxicação aguda \\
\hline F18.0 & Uso de solventes voláteis - intoxicação aguda \\
\hline F19.0 & Uso de múltiplas drogas e outras substâncias psicoativas - intoxicação aguda \\
\hline \multicolumn{2}{|r|}{ Capítulo XVI: Algumas Afecções Originadas no Período Perinatal } \\
\hline P93 & Reações e intoxicações devidas a drogas administradas ao feto e ao recém-nascido \\
\hline \multicolumn{2}{|r|}{ Capítulo XX: Causas Externas de Morbidade e de Mortalidade } \\
\hline \multicolumn{2}{|c|}{ Contato com Animais e Plantas Venenosos } \\
\hline $\mathrm{X} 28$ & Contato com outras plantas venenosas especificadas \\
\hline \multicolumn{2}{|c|}{ Envenenamento [Intoxicação] Acidental por e Exposição a Substâncias Nocivas } \\
\hline $\mathrm{X} 40$ & Analgésicos, antipiréticos e antirreumáticos, não opiáceos \\
\hline $\mathrm{X} 41$ & $\begin{array}{l}\text { Anticonvulsivantes [antiepilépticos], sedativos, hipnóticos, antiparkinsonianos e } \\
\text { psicotrópicos, não classificados em outra parte }\end{array}$ \\
\hline $\mathrm{X} 42$ & Narcóticos e psicodislépticos [alucinógenos], não classificados em outra parte \\
\hline $\mathrm{X} 43$ & Outras substâncias farmacológicas de ação sobre o sistema nervoso autônomo \\
\hline $\mathrm{X} 44$ & Outras drogas, medicamentos e substâncias biológicas não especificadas \\
\hline $\mathrm{X} 45$ & Exposição ao álcool \\
\hline $\mathrm{X} 46$ & Solventes orgânicos e hidrocarbonetos halogenados e seus vapores \\
\hline $\mathrm{X} 47$ & Outros gases e vapores \\
\hline $\mathrm{X} 48$ & Pesticidas \\
\hline $\mathrm{X} 49$ & Outras substâncias químicas nocivas e às não especificadas \\
\hline
\end{tabular}




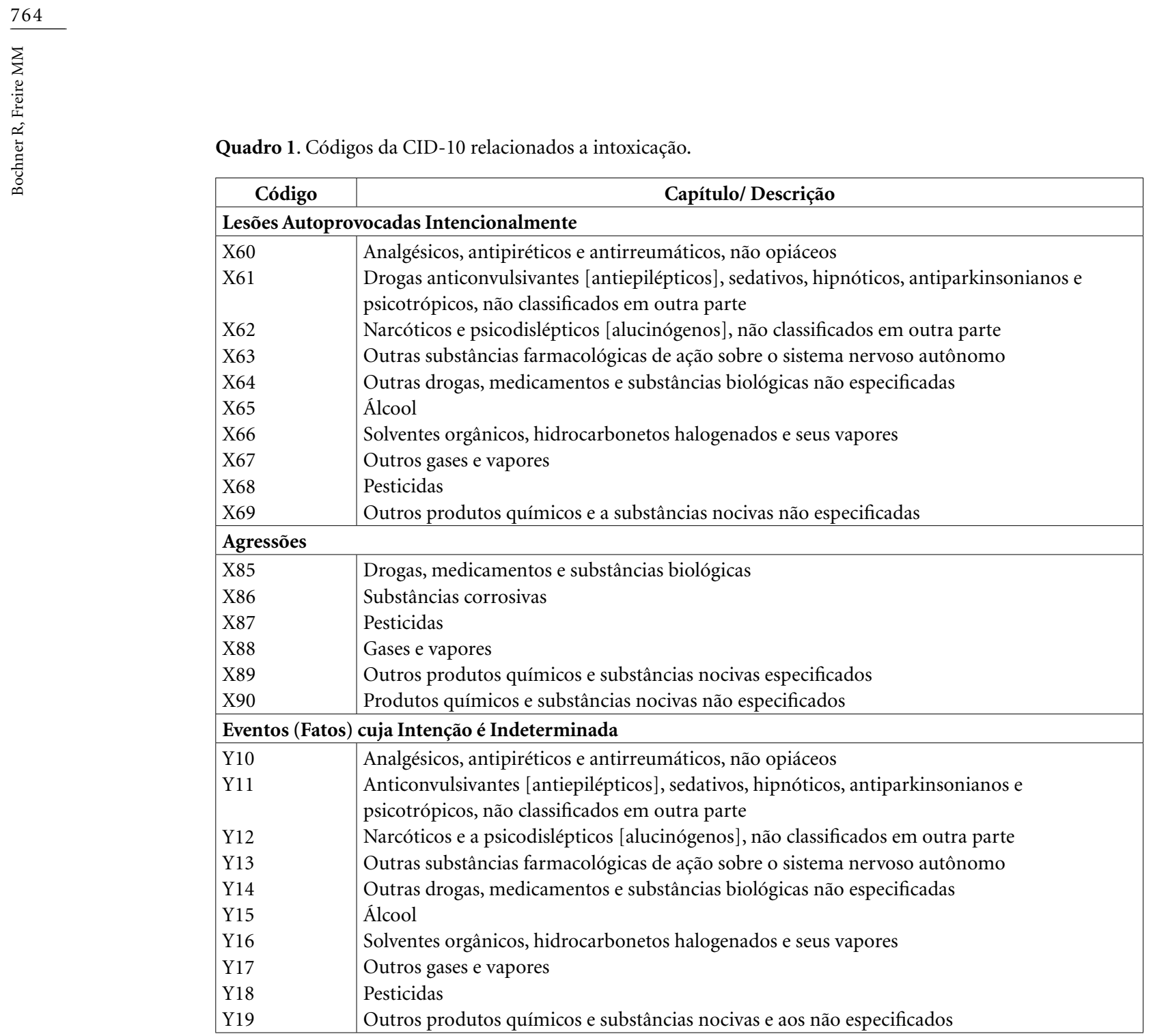

A variável circunstância utilizada neste estudo foi construída com base nos códigos da CID10 presentes na variável causa básica, da seguinte forma: acidental - X28 e X40 a X49; suicídio X60 a X69; agressão - X85 a X90; não determinada - Y10 a Y19; não classificada - A02, A05, F10.0 a F19.0, P93.

Foram calculados os coeficientes de mortalidade por 100.000 habitantes para as regiões geográficas referente ao período de 2010 a 2015, padronizadas por faixa etária e sexo pela população do Brasil em 2010, utilizando as estimativas populacionais fornecidas pelo DATASUS ${ }^{11}$.

Os APVP foram determinados pela soma das diferenças entre as idades individuais das vítimas e as expectativas anuais de vida por sexo disponibilizadas pelo Instituto Brasileiro de Geografia e Estatística (IBGE) ${ }^{12}$. Para o sexo masculino con- siderou-se 70 anos em 2010 e 2011, 71 em 2012, 2013 e 2014 e 72 em 2015. Para o sexo feminino, nos anos de 2010 e 2011 adotou-se a expectativa de vida de 77 anos, para os anos de 2012, 2013 e 2014 o valor considerado foi de 78 anos e para 2015 adotou-se o valor de 79 anos.

Para análise de tendência, foi aplicada regressão exponencial para todos os agentes tóxicos.

Este estudo foi aprovado pelo Comitê de Ética em Pesquisa da Escola Politécnica de Saúde Joaquim Venâncio, unidade da Fundação Oswaldo Cruz.

\section{Resultados}

No Brasil, no período de 2010 a 2015, foram registrados pelo SIM 18.247 óbitos decorrentes de 
intoxicação, resultando em um crescimento de $3 \%$ no coeficiente de mortalidade.

Dentre os nove agentes tóxicos identificados pelos códigos da CID-10, os agrotóxicos, as drogas de abuso e os medicamentos figuraram como os principais agentes responsáveis pelos óbitos, tanto para as regiões geográficas quanto para o país como um todo. Nas regiões Nordeste e Centro-Oeste, assim como no Brasil, destacaram-se os agrotóxicos. Na região Norte, as drogas de abuso foram apontadas como o principal agente tóxico e nas regiões Sul e Sudeste os medicamentos assumiram a liderança.
A Tabela 1 apresenta, para o período de 2010 a 2015, o número de óbitos por intoxicação e coeficientes de mortalidade por 100.000 habitantes distribuídos por sexo, agente tóxico, faixa etária e região geográfica. Para a região os coeficientes foram padronizados por sexo e faixa etária com base na população do país em 2010.

No Brasil, no período de 2010 a 2015, a participação do sexo masculino nos óbitos por intoxicação foi de $66 \%$. O coeficiente de mortalidade para intoxicação foi de 1,52 por 100.000 habitantes, sendo 1,01 para o sexo feminino e 2,05 para o masculino, o que demonstra um risco duas vezes

Tabela 1. Número (N) de óbitos por intoxicação, proporção (\%) e coeficiente de mortalidade por 100.000 habitantes segundo agente tóxico, sexo, faixa etária e região geográfica. Brasil, 2010 a 2015.

\begin{tabular}{|c|c|c|c|c|c|c|c|c|c|}
\hline \multirow{3}{*}{ Variáveis } & \multicolumn{6}{|c|}{ Sexo } & \multirow{2}{*}{\multicolumn{3}{|c|}{ Total }} \\
\hline & \multicolumn{3}{|c|}{ Masculino } & \multicolumn{3}{|c|}{ Feminino } & & & \\
\hline & $\mathbf{N}$ & $\%$ & Coef. & $\mathbf{N}$ & $\%$ & Coef. & $\mathbf{N}$ & $\%$ & Coef. \\
\hline \multicolumn{10}{|l|}{ Agente tóxico } \\
\hline Agrotóxico & 2878 & 23,7 & 0,49 & 1503 & 24,6 & 0,25 & 4381 & 24,0 & 0,36 \\
\hline Medicamento & 1978 & 16,3 & 0,33 & 2142 & 35,1 & 0,35 & 4120 & 22,6 & 0,34 \\
\hline Droga de abuso & 3287 & 27,1 & 0,55 & 640 & 10,5 & 0,11 & 3927 & 21,5 & 0,33 \\
\hline Produto químico industrial & 798 & 6,6 & 0,13 & 254 & 4,2 & 0,04 & 1052 & 5,8 & 0,09 \\
\hline Alimento & 173 & 1,4 & 0,03 & 153 & 2,5 & 0,03 & 326 & 1,8 & 0,03 \\
\hline Droga de abuso + Prod. quím.ind. & 932 & 7,7 & 0,16 & 164 & 2,7 & 0,03 & 1096 & 6,0 & 0,09 \\
\hline Droga de abuso + Medicamento & 54 & 0,4 & 0,01 & 18 & 0,3 & 0,00 & 72 & 0,4 & 0,01 \\
\hline Planta & 8 & 0,1 & 0,00 & 6 & 0,1 & 0,00 & 14 & 0,1 & 0,00 \\
\hline Miscelânea ${ }^{\star}$ & 2028 & 16,7 & 0,34 & 1228 & 20,1 & 0,20 & 3256 & 17,8 & 0,27 \\
\hline \multicolumn{10}{|l|}{ Faixa Etária } \\
\hline 0 a 4 & 147 & 1,2 & 0,31 & 121 & 2,0 & 0,27 & 268 & 1,5 & 0,29 \\
\hline 5 a 9 & 35 & 0,3 & 0,07 & 17 & 0,3 & 0,04 & 52 & 0,3 & 0,05 \\
\hline 10 a 14 & 84 & 0,7 & 0,16 & 137 & 2,2 & 0,27 & 221 & 1,2 & 0,22 \\
\hline 15 a 19 & 962 & 7,9 & 1,84 & 533 & 8,7 & 1,05 & 1495 & 8,2 & 1,45 \\
\hline 20 a 29 & 2646 & 21,8 & 2,52 & 1049 & 17,2 & 1,02 & 3695 & 20,3 & 1,77 \\
\hline 30 a 39 & 2591 & 21,3 & 2,72 & 1190 & 19,5 & 1,24 & 3781 & 20,7 & 1,98 \\
\hline 40 a 49 & 2302 & 19,0 & 3,02 & 1257 & 20,6 & 1,59 & 3559 & 19,5 & 2,29 \\
\hline 50 a 59 & 1601 & 13,2 & 2,78 & 858 & 14,0 & 1,38 & 2459 & 13,5 & 2,05 \\
\hline 60 a 69 & 865 & 7,1 & 2,54 & 448 & 7,3 & 1,13 & 1313 & 7,2 & 1,78 \\
\hline 70 a 79 & 563 & 4,6 & 3,38 & 277 & 4,5 & 1,26 & 840 & 4,6 & 2,17 \\
\hline $80 \mathrm{e}+$ & 279 & 2,3 & 4,12 & 214 & 3,5 & 1,92 & 493 & 2,7 & 2,75 \\
\hline Ign & 61 & 0,5 & & 7 & 0,1 & & 68 & 0,4 & \\
\hline \multicolumn{10}{|l|}{ Região } \\
\hline Norte & 1139 & 9,4 & 2,35 & 385 & 6,3 & 0,81 & 1524 & 25,0 & 1,57 \\
\hline Nordeste & 3254 & 26,8 & 2,04 & 1629 & 26,7 & 0,98 & 4883 & 79,9 & 1,50 \\
\hline Sudeste & 5316 & 43,8 & 2,04 & 2700 & 44,2 & 1,00 & 8016 & 131,2 & 1,51 \\
\hline Sul & 1397 & 11,5 & 1,54 & 843 & 13,8 & 0,92 & 2240 & 36,7 & 1,23 \\
\hline Centro-Oeste & 1030 & 8,5 & 2,26 & 551 & 9,0 & 1,23 & 1581 & 25,9 & 1,74 \\
\hline Total & 12136 & 100,0 & 2,04 & 6108 & 100,0 & 1,01 & 18244 & 100,0 & 1,52 \\
\hline
\end{tabular}

Fonte: Sistema de Informação de Mortalidade (SIM).

Excluído 3 registros com sexo ignorado. * prod. quím ind. + domissanitário + plantas + metais + alimentos 
maior para os homens de morrer em decorrência de intoxicações quando comparado às mulheres (Tabela 1).

Segundo o agente tóxico envolvido, observase que os coeficientes de mortalidade por agrotóxicos, medicamentos e drogas de abuso, 0,36; 0,34 e 0,33 respectivamente, foram os maiores quando comparados aos demais agentes. Além disso, esses três agentes concentraram $68,1 \%$ dos óbitos (Tabela 1).

Grandes diferenças entre os sexos são observadas para os coeficientes de mortalidade por drogas de abuso e por agrotóxicos. O risco de morrer em decorrência de intoxicação por drogas de abuso foi 5 vezes maior entre homens do que entre mulheres. Já para os agrotóxicos, o risco entre homens foi duas vezes maior que o observado entre mulheres. Os homens apresentaram maior concentração de óbitos por drogas de abuso e agrotóxicos (51\%), enquanto que para as mulheres os medicamentos e agrotóxicos responderam pela maioria dos óbitos (60\%) (Tabela 1).

Com relação às faixas etárias mais acometidas, $61 \%$ estão relacionados a adultos com idades entre 20 a 49 anos.

Os maiores coeficientes de mortalidade foram observados em adultos de 40 a 49 anos e idosos com 80 anos ou mais. Esse mesmo comportamento é observado para o sexo feminino, contudo para o sexo masculino os maiores valores de coeficientes de mortalidade encontram-se nas faixas de 70 a 79 anos e de 80 anos e mais. Com exceção da faixa de 10 a 14 anos, para todas as demais faixas etárias os coeficientes de mortalidade para o sexo masculino foram superiores ao do sexo feminino, implicando em riscos maiores para homens, variando de 1,2 a 2,7 vezes, quando comparados aos das mulheres (Tabela 1).

A análise segundo as regiões geográficas demonstrou ser mais homogênea do que as avaliações segundo sexo e faixa etária. A região Centro-Oeste apresentou o maior valor, 1,74 por 100.000 habitantes. As taxas de mortalidade para o sexo masculino mostraram-se superiores ao do sexo feminino em todas as regiões, contudo com riscos diferenciados. Na Região Norte, o risco de um homem morrer em decorrência de intoxicação é 2,9 vezes maior do que o de uma mulher. Nas demais regiões esse risco é de 2,1 para a região Nordeste; 2,0 para a Sudeste; 1,7 para a Sul e 1,8 para a Centro-Oeste (Tabela 1 ).

A Tabela 2 apresenta a distribuição por agente tóxico e ano dos óbitos decorrentes de intoxicação registrados pelo SIM para os anos de 2010 a 2015. Fornece também o coeficiente de mor- talidade padronizado por faixa etária e sexo e o total de APVP.

Com base na análise temporal dos coeficientes padronizados para os anos de 2010 a 2015, verificou-se tendência significativa de decréscimo para agrotóxicos (p-valor 0,004), alimentos (p-valor 0,036) e miscelânea (p-valor 0,032), e aumento para as drogas de abuso ( $\mathrm{p}$-valor 0,029), produtos químicos industriais (p-valor 0,012 ) e drogas de abuso + produtos químicos ( $\mathrm{p}$-valor 0,021).

Ao analisar os 18.247 óbitos por intoxicação, verificou-se um total de 608.059 APVP, o que representa uma média de 33 anos potenciais de vida perdidos por óbito. No período do estudo observou-se um crescimento nos APVP de $18 \%$. As drogas de abuso foram responsáveis por $24 \%$ do total dos APVP, superando os agrotóxicos e os medicamentos, ambos com 22\% (Tabela 2).

Ao longo do período do estudo verificou-se um aumento importante dos óbitos por drogas de abuso, que chegaram a ultrapassar o número de óbitos por medicamentos nos anos de 2011 e 2013 e também o número por agrotóxicos nos anos de 2013, 2014 e 2015. Este aumento é ainda mais pronunciado ao levar em consideração os APVP, quando observa-se que esse indicador é maior para drogas de abuso do que para medicamentos em 2011, 2013, 2014 e 2015 e maior do que agrotóxicos em 2012, 2013, 2014 e 2015 (Tabela 2).

A Figura 1 apresenta a distribuição por faixa etária e sexo para os principais agentes tóxicos envolvidos nos óbitos decorrentes de intoxicação: medicamentos, agrotóxicos, drogas de abuso e produtos químicos industriais.

Com exceção dos medicamentos, em que a participação do sexo feminino nos óbitos é de $52 \%$, verifica-se maior concentração de óbitos para o sexo masculino em todos os agentes: drogas de abuso (84\%), produtos químicos industriais $(76 \%)$ e agrotóxicos $(65 \%)$, bem como para a maioria das faixas etárias. Somente para agrotóxicos e medicamentos nas faixas etárias de 10 a 14 e 15 a 19 anos e produtos químicos industriais nas faixas de 0 a 4 e 10 a 14 anos foram observadas maior participação do sexo feminino nos óbitos (Figura 1).

A análise do formato da distribuição por faixa etária dos óbitos demonstra similaridade entre pares de agentes tóxicos. Os medicamentos e agrotóxicos apresentam uma distribuição aproximadamente simétrica em torno da faixa de 40 a 49 anos. As drogas de abuso e produtos químicos industriais apresentam uma distribuição assi- 
Tabela 2. Distribuição de óbitos registrados pelo SIM, coeficiente de mortalidade padronizado por faixa etária e sexo, APVP, por agente tóxico e ano. Brasil, 2010 a 2015.

\begin{tabular}{|c|c|c|c|c|c|c|c|c|}
\hline \multicolumn{2}{|c|}{ Agentes tóxicos } & \multirow{2}{*}{$\begin{array}{r}2010 \\
897\end{array}$} & \multirow{2}{*}{$\begin{array}{r}2011 \\
891\end{array}$} & \multirow{2}{*}{$\begin{array}{c}2012 \\
694\end{array}$} & \multirow{2}{*}{$\begin{array}{r}2013 \\
672\end{array}$} & \multirow{2}{*}{$\begin{array}{r}2014 \\
607\end{array}$} & \multirow{2}{*}{$\begin{array}{r}2015 \\
620\end{array}$} & \multirow{2}{*}{$\begin{array}{r}\text { Total } \\
4381\end{array}$} \\
\hline Agrotóxicos & Óbitos & & & & & & & \\
\hline & Coef & 0,46 & 0,45 & 0,34 & 0,32 & 0,29 & 0,29 & 0,36 \\
\hline & APVP & 28545 & 27608 & 21966 & 20844 & 18013 & 19689 & 136665 \\
\hline \multirow[t]{3}{*}{ Medicamentos } & Óbitos & 652 & 649 & 734 & 648 & 726 & 711 & 4120 \\
\hline & Coef & 0,33 & 0,33 & 0,36 & 0,32 & 0,35 & 0,34 & 0,34 \\
\hline & APVP & 21472 & 20984 & 23720 & 20936 & 24514 & 23831 & 135457 \\
\hline \multirow[t]{3}{*}{ Drogas de abuso } & Óbitos & 508 & 659 & 627 & 677 & 709 & 747 & 3927 \\
\hline & Coef & 0,26 & 0,33 & 0,31 & 0,33 & 0,35 & 0,36 & 0,32 \\
\hline & APVP & 16089 & 21630 & 23555 & 26226 & 28183 & 30038 & 145721 \\
\hline \multirow{3}{*}{$\begin{array}{l}\text { Prod. Quím. } \\
\text { Industriais }\end{array}$} & Óbitos & 102 & 88 & 138 & 196 & 276 & 253 & 1053 \\
\hline & Coef & 0,05 & 0,04 & 0,07 & 0,10 & 0,14 & 0,12 & 0,09 \\
\hline & APVP & 3137 & 2819 & 4736 & 7441 & 12031 & 10965 & 41129 \\
\hline \multirow[t]{3}{*}{ Alimentos } & Óbitos & 70 & 68 & 48 & 45 & 52 & 43 & 326 \\
\hline & Coef & 0,04 & 0,03 & 0,02 & 0,02 & 0,02 & 0,02 & 0,02 \\
\hline & APVP & 2241 & 1918 & 1370 & 1255 & 1436 & 1034 & 9254 \\
\hline \multirow{3}{*}{$\begin{array}{l}\text { Drogas de abuso + } \\
\text { Prod. Quím. } \\
\text { Industriais }\end{array}$} & Óbitos & 81 & 128 & 176 & 261 & 225 & 226 & 1097 \\
\hline & Coef & 0,04 & 0,06 & 0,08 & 0,13 & 0,11 & 0,11 & 0,09 \\
\hline & APVP & 2545 & 3730 & 4707 & 7062 & 6277 & 6586 & 30907 \\
\hline \multirow{3}{*}{$\begin{array}{l}\text { Drogas }+ \\
\text { Medicamentos }\end{array}$} & Óbitos & 13 & 12 & 9 & 15 & 15 & 8 & 72 \\
\hline & Coef & 0,01 & 0,01 & 0,00 & 0,01 & 0,01 & 0,00 & 0,01 \\
\hline & APVP & 531 & 441 & 381 & 546 & 624 & 347 & 2870 \\
\hline \multirow[t]{3}{*}{ Plantas } & Óbitos & - & 1 & 2 & 2 & 4 & 5 & 14 \\
\hline & Coef & - & 0,00 & 0,00 & 0,00 & 0,00 & 0,00 & 0,00 \\
\hline & APVP & - & 15 & 63 & 80 & 142 & 164 & 464 \\
\hline \multirow[t]{3}{*}{ Miscelânea } & Óbitos & 584 & 561 & 573 & 483 & 534 & 522 & 3257 \\
\hline & Coef & 0,30 & 0,28 & 0,28 & 0,23 & 0,25 & 0,24 & 0,25 \\
\hline & APVP & 18616 & 18004 & 19465 & 15189 & 17240 & 17078 & 105592 \\
\hline \multirow[t]{3}{*}{ Total } & Óbitos & 2907 & 3057 & 3001 & 2999 & 3148 & 3135 & 18247 \\
\hline & Coef & 1,48 & 1,53 & 1,48 & 1,46 & 1,52 & 1,49 & 1,49 \\
\hline & APVP & 93176 & 97149 & 99963 & 99579 & 108460 & 109732 & 608059 \\
\hline
\end{tabular}

métrica à direita, diferindo apenas pelo fato das drogas de abuso praticamente não apresentarem óbitos para as faixas de 0 a 4 e de 5 a 9 anos. Apenas um óbito na faixa etária de 0 a 4 anos, para o sexo masculino, foi registrado no período do estudo por drogas de abuso (Figura 1).

Para medicamentos e agrotóxicos o suicídio foi a principal circunstância dos óbitos, respondendo por $58 \%$ e $78 \%$, respectivamente. Já para as drogas de abuso e produtos químicos industriais a circunstância acidental foi a que concentrou o maior percentual de óbitos, $49 \%$ e $53 \%$, respectivamente.

Para os medicamentos, os códigos X44, X64 e Y14 que contemplam outras drogas, medicamentos e substâncias biológicas não especifica- das concentraram 50\% dos óbitos; seguidos dos anticonvulsivantes [antiepilépticos], sedativos, hipnóticos, antiparkinsonianos e psicotrópicos, representados nos códigos X41, X61 e Y11, com 39\% dos óbitos.

Para as drogas de abuso, os narcóticos e psicodislépticos [alucinógenos], descritos nos códigos X42, X62 e Y12, e o uso de álcool, traduzido pelo código F10.0, responderam por quase a totalidade dos óbitos, $67 \%$ e $27 \%$, respectivamente.

Para os produtos químicos industriais, outros gases e vapores presentes nos códigos X47, X67, Y17 e X88, e os solventes orgânicos e hidrocarbonetos halogenados e seus vapores, representados pelos códigos X46, X66, Y16, concentraram 40\% e $44 \%$ dos óbitos, respectivamente. 


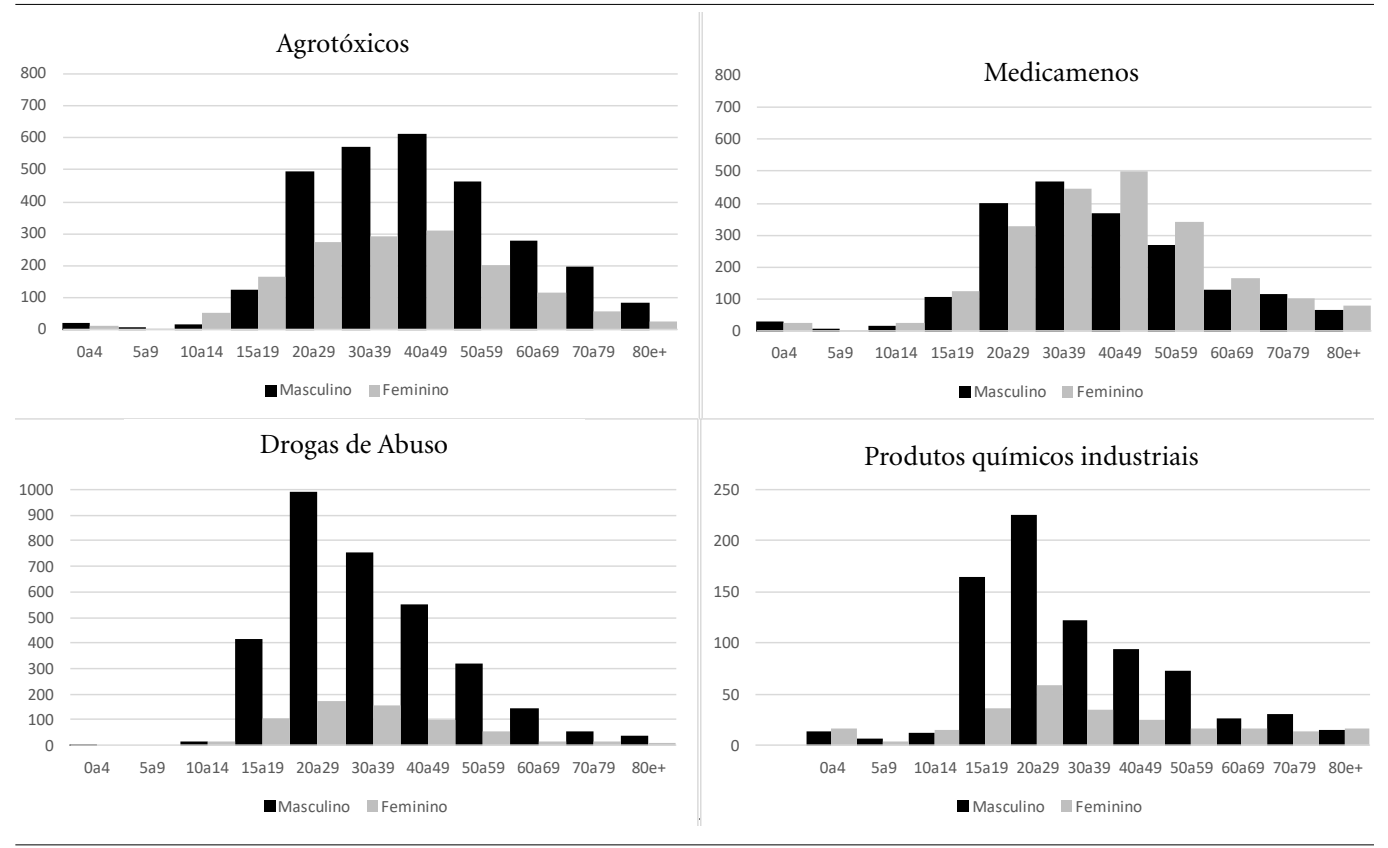

Figura 1. Distribuição por faixa etária e sexo para medicamentos, agrotóxicos, drogas de abuso, e produtos químicos industriais. Brasil, 2010 a 2015.

A circunstância agressão não foi elencada para os medicamentos e para as drogas de abuso pelo fato do código da CID-10, X85, reunir esses dois agentes para avaliar essa circunstância.

Com relação a variável escolaridade, $20 \%$ dos registros não foram preenchidos ou são ignorados. Assim, partindo de um total de 14.524, a distribuição de escolaridade das vítimas deu-se da seguinte forma: $34,5 \%$ possuíam até 3 anos de estudo, $33,7 \%$ tinham 4 a 7 anos de estudo; $23,9 \%$ apresentavam 8 a 11 anos de estudo e $7,9 \%$ possuíam mais de 12 anos de escolaridade. Esse perfil, com maior percentual de óbitos em vítimas com baixa escolaridade foi observado para agrotóxicos e medicamentos, $37,3 \%$ e $30,6 \%$, respectivamente. Para os demais agentes tóxicos a maioria das vítimas apresentaram 4 a 7 anos de estudo: drogas de abuso (40\%) e produtos químicos industriais $(36,8 \%)$.

\section{Discussão}

No Brasil, os óbitos por intoxicações vitimaram predominantemente indivíduos do sexo masculino, fato relacionado às diferenças nas características de saúde entre os sexos, bem como daquelas ligadas ao gênero, que levam em consideração mudanças comportamentais ${ }^{10}$.
A maioria dos indicadores tradicionais de saúde mostra, com clareza, a existência de diferencial entre os sexos, sendo a mortalidade masculina maior em praticamente todas as idades ${ }^{13}$.

Ao analisar a mortalidade decorrente de intoxicação por sexo e faixa etária, as taxas para o sexo masculino mostraram-se superiores às do sexo feminino. Somente os pré-adolescentes, representados pela faixa de 10 a 14 anos, apresentaram taxa de mortalidade superior para o sexo feminino em comparação ao masculino. Esses pré-adolescentes se encontram em uma etapa de transição localizada entre a infância e a adolescência/início da vida adulta ${ }^{14}$. Nessa etapa, com relação aos óbitos decorrentes de intoxicação, os meninos costumam apresentar comportamento mais próximo da faixa etária de 5 a 9 anos, na qual prevalece a circunstância acidental. Por outro lado, as meninas mostram um perfil que se aproxima das jovens de 15 a 19 anos, para as quais o suicídio aparece como principal circunstância ${ }^{15}$. Sabe-se que a letalidade das tentativas de suicídio é maior que a dos acidentes. Estes fatos podem explicar o menor número de óbitos do sexo masculino na faixa de 10 a 14 anos quando comparados ao do sexo feminino.

As intoxicações geram óbitos concentrados na faixa etária de 20 a 49 anos, o que impõe um número alto de APVP, indicador que incorpora 
informações sobre o impacto social e econômico da morte ${ }^{16}$.

Os resultados apresentados foram capazes de delinear um perfil de mortalidade para cada um dos agentes tóxicos estudados.

Os óbitos causados por agrotóxicos ocorreram em sua maioria em homens (65\%), na faixa etária de 30 a 49 anos (40\%), com baixa escolaridade ( $<=3$ anos) (36\%), pela circunstância suicídio (79\%). Esse perfil de mortalidade acompanha o de morbidade já descrito em outros estudos ${ }^{17,18}$. A baixa escolaridade observada mostrou-se em consonância com Soares et al. ${ }^{19}$, uma vez que esses autores consideraram a escolaridade um fator de proteção à intoxicação por agrotóxicos.

O maior número de óbitos causados por agrotóxicos já é um resultado recorrente nas estatísticas dos sistemas nacionais de informação ${ }^{20,21}$, explicado por suas altas toxicidade e letalidade comparadas aos demais agentes ${ }^{22,23}$. Além disso, o Brasil passou a ocupar desde 2009, o primeiro lugar no ranking mundial de consumo de agrotóxi$\cos ^{24}$. Diante disso, a tendência de diminuição do coeficiente de mortalidade por agrotóxicos observada neste estudo deve ser encarada com ressalvas, uma vez que as políticas do uso de agrotóxicos no país não mudaram, pelo contrário, estão cada vez mais intensas ${ }^{22}$. Deve-se considerar também a subnotificação de óbitos decorrentes de intoxicação por agrotóxicos já apontada por Bochner $^{25}$, resultante do preenchimento equivocado da causa básica do óbito.

Apesar da baixa toxicidade dos medicamentos, traduzida pelos seus baixos valores de letalidade $^{23,26}$, estes figuram como o segundo principal agente tóxico responsável pelos óbitos de intoxicação. Nesse sentido, a magnitude dos casos de intoxicação por medicamentos, principal agente tóxico para os casos de intoxicação ${ }^{20,21,23,26}$, justifica esse achado.

Os óbitos decorrentes de intoxicação por medicamentos referem-se em sua maioria a mulheres (52\%), na faixa etária de 30 a 49 anos (43\%), com 4 a 7 anos de escolaridade, pela circunstância suicídio. A relação de medicamentos com o sexo feminino, bem como a participação da faixa etária de 30 a 49 anos, já foi apontada por outros estudos ${ }^{26-29}$.

Os óbitos por intoxicações, causados por medicamento, acometem de forma significativa crianças menores de 5 anos, em circunstância acidental. Bortoletto e Bochner ${ }^{26}$, em 1999, já apontavam para a preocupação com essas intoxicações, ressaltando que crianças nesta faixa etária são as maiores vítimas de intoxicações por esse agente toxico no país. Até os 5 anos de idade, as crianças estão desenvolvendo suas habilidades motoras, descobrindo o novo, principalmente através do toque. Esta característica comportamental das crianças torna-se preocupante em países como o Brasil, onde há um padrão de consumo de medicamentos descontrolado, com facilidade de compra e consequente grande disponibilidade de medicamentos nas residências, possibilitando o contato de crianças com esses agentes tóxicos. Além disso, não temos exigência legal bem fundamentada para embalagens seguras de produtos farmacêuticos, fato que favorece as intoxicações e óbitos por medicamentos em crianças na faixa etária abaixo dos $5 \operatorname{anos}^{30}$.

Apesar de agrotóxicos e medicamentos apresentarem uma distribuição por faixa etária similar e a mesma principal circunstância, o suicídio, cabe salientar as diferenças encontradas entre esses dois agentes, que impõem formas distintas de enfrentamento. Por um lado, os agrotóxicos com maior participação do sexo masculino e baixa escolaridade, e do outro, os medicamentos com maior participação do sexo feminino e maior nível de escolaridade, ambos exigindo políticas públicas específicas focadas em suas particularidades.

Entre as classes de medicamentos mais envolvidas com óbitos estão os anticonvulsivantes [antiepilépticos], sedativos, hipnóticos, antiparkinsonianos e psicotrópicos. Esses grupos farmacológicos foram também elencados por Bernardes et al. ${ }^{27}$ ao analisar o perfil das tentativas de suicídio atendidas pelo Centro de Controle de Intoxicações de Londrina, no período de 1997 a 2007. Esse comportamento pode ser explicado pelo fato desses medicamentos possuírem várias indicações terapêuticas, sofrerem prescrição médica indiscriminada, além de serem de baixo custo. Tudo isso favorece ainda mais o acesso a esses produtos e consequentemente a automedicação.

Os óbitos decorrentes de intoxicação por drogas de abuso ocorrem principalmente em pessoas do sexo masculino (84\%), na faixa etária de 20 a 39 anos (52\%), com 4 a 7 anos de estudo (40\%), pela circunstância acidental (45\%).

Em número de óbitos, as drogas ficam em terceiro lugar, entretanto ocupam a segunda posição quando se avalia os APVP, mostrando que esse agente acomete vítimas mais jovens do que as de medicamentos, mesmo não apresentando incidência em crianças.

$\mathrm{O}$ aumento dos óbitos por drogas de abuso seria ainda mais pronunciado se os códigos da CID-10 permitissem a separação, em alguns ca- 
sos, das drogas de medicamentos (X85) e também de produtos químicos industriais (X45, X65, Y15). Em especial o álcool utilizado como bebida ficou subestimado por estar misturado com outros tipos de álcoois, estes pertencentes a categoria dos produtos químicos industriais. Ainda assim, neste estudo, o álcool esteve envolvido em $30 \%$ dos óbitos decorrentes de intoxicação por drogas de abuso. Outros trabalhos já sinalizaram a importância do álcool devido ao seu grande consumo, em especial pelos jovens, o que o torna um forte agente causador de intoxicações ${ }^{31-34}$.

Os óbitos decorrentes de drogas de abuso, apesar de subdimensionados por problemas de classificação da CID-10, merecem destaque tanto pelo crescimento observado no período do estudo, quanto pelo elevado número de APVP, evidenciado pela sua distribuição por faixa etária, concentrada em jovens, muito diferente da curva para os óbitos em geral. Dentre as drogas envolvidas, o álcool é a principal substância. O grande aumento dos óbitos decorrentes de intoxicação por drogas pode ter relação com o uso concomitante e abusivo de álcool e bebidas energéticas a base de cafeína, taurina e carboidratos, por parte da população, em especial de jovens. O uso de bebidas energéticas está diretamente relacionado ao consumo de álcool. Ferreira, já em 2004, apontava para a rápida popularização da ingestão de bebidas alcoólicas, principalmente destiladas, em combinação com bebidas energéticas ${ }^{35}$.

Além disso, outro estudo realizado em 2010 com universitários nos EUA, avaliou o aumento do consumo de álcool, drogas e medicamentos associado ao consumo de bebidas energéticas. Os resultados mostraram que em comparação com os jovens que não consumiam bebidas energéticas, aqueles que faziam uso delas tinham padrões de maior consumo de álcool e estavam mais propensos ao uso de drogas e medicamentos ${ }^{36}$.

Os óbitos decorrentes de produtos químicos industriais ocorreram em sua maioria em homens (76\%), na faixa etária de 15 a 29 anos (42\%), com 4 a 7 anos de estudo (36\%) pela circunstância acidental (48\%).

Apesar da maioria dos óbitos decorrentes de intoxicação estar relacionada com suicídio, é importante salientar que para drogas de abu- so e produtos químicos industriais essa relação não foi observada. Nesses casos os acidentes são a principal circunstância, o que impõe medidas diferenciadas voltadas a prevenção e controle.

A relação entre drogas de abuso e produtos químicos industriais observada pelas suas curvas de distribuição de faixa etária, somado com as similaridades do uso abusivo que é feito dos produtos químicos ${ }^{15}$, indicam que mais estudos devem ser realizados para esses dois agentes tóxicos. É importante salientar que para os produtos químicos há incidência de óbitos em crianças, o que é raro de observar em se tratando de drogas de abuso. Nesse sentido muitos afirmam que os produtos químicos, como cola de sapateiro, benzina, tiner, e outros solventes, são uma das portas de entrada para o consumo das drogas ${ }^{33,37}$. No entanto, não se pode esquecer que muitos desses produtos encontram-se dentro das residências, o que favorece a ocorrência de acidentes envolvendo crianças.

Os maiores crescimentos de mortalidade decorrentes de intoxicação observados no período ocorreram para esses dois agentes e a combinação entre eles, drogas de abuso + produtos químicos industriais (178\%), produtos químicos industriais (171\%) e drogas de abuso (40\%). Tal constatação reforça a necessidade da realização de estudos mais aprofundados sobre esses agentes.

\section{Conclusões}

A análise conjunta dos agentes tóxicos envolvidos nos óbitos nacionais decorrentes de intoxicação possibilitou uma avaliação comparativa do perfil da mortalidade relacionada a cada um desses agentes, bem como foi possível verificar suas tendências de aumento ou diminuição para o período do estudo. Esta análise ampla e exploratória mostrou a participação expressiva e crescente das drogas de abuso nos óbitos, em especial evidenciada pelos APVP.

Outro ponto importante que deve ser ressaltado é a dificuldade de se trabalhar com os óbitos por intoxicação utilizando a CID-10, devido à classificação toxicologicamente pouco qualificada dos agentes tóxicos, gerando perdas de casos e dados menos confiáveis. 


\section{Colaboradores}

R Bochner trabalhou na concepção do estudo, coleta e análise dos dados, redação e revisão final do artigo. MM Freire trabalhou na pesquisa bibliográfica, metodologia, análise dos dados e redação do artigo.

\section{Referências}

1. Trevisol FS, Custódio G, Locks LH, Trevisol DJ. Avaliação das mortes por causas externas na cidade de Tubarão (SC) no ano de 2009. Rev. Assoc. Méd. Rio Gd. Sul 2011; 55(1):25-30.

2. World Health Organization (WHO). International Programme on Chemical Safety (IPCS) [homepage na internet]. Poisoning Prevention and Management. [acessado 2018 Jan 2]. Disponível em: http://www. who.int/ipcs/poisons/en/.

3. Zambolim CM, Oliveira TP, Hoffmann AN, Vilela CEB, Neves D, Anjos FR, Soares LM, Tiburzio LS, Cardoso LAF, Murad MB, Magalhães MG, Oppermann PER, Guimarães SJ. Perfil das intoxicações exógenas em um hospital universitário. Rev. Méd. Minas Gerais 2018; 18(1):5-10.

4. Laurenti R, Jorge MHPM, Gotlieb SLD. Estatísticas de mortalidade e seus usos. R. Eletr. de Com. Inf. Inov. Saúde 2013; 7(2).

5. Silva MGC. Anos potenciais de vida perdidos por causas evitáveis, segundo sexo, em Fortaleza, em 19961998. Epidemiol. Serv. Saúde 2003; 12(2):99-110.

6. Reichenheim ME, Werneck GL. Anos potenciais de vida perdidos no Rio de Janeiro, 1990. As mortes violentas em questão. Cad Saude Publica 1994; 10(Supl. 1):188-198.

7. Mota DM, Melo JRR, Freitas DRC, Machado M. Perfil da mortalidade por intoxicação com medicamentos no Brasil, 1996-2005: retrato de uma década. Cien Saude Colet 2012; 17(1):61-70.

8. Santana VS, Moura MCP, Nogueira FF. Mortalidade por intoxicação ocupacional relacionada a agrotóxicos, 2000-2009, Brasil. Rev Saude Publica 2013; 47(3):598-606.

9. Brasil. Ministério da Saúde (MS). Departamento de Informática. DATASUS. [homepage na internet]. Acesso à Informação. Serviços. Transferência de Arquivos. SIM. Arquivos de Dados. DO - Declarações de Óbito. 1979 a 2015. [acessado 2016 Set 4]. Disponível em: http://www2.datasus.gov.br/DATASUS/index.php?area $=0901 \&$ item $=1 \&$ acao $=26 \&$ pad $=31655$

10. Organização Mundial da Saúde (OMS). Classificação Estatística Internacional de Doenças e Problemas Relacionados à Saúde. São Paulo: Edusp; 1995. Vol 1.

11. Brasil. Ministério da Saúde (MS). Departamento de Informática. DATASUS. [homepage na internet]. Acesso à Informação. TABNET. Demográficas e Socioeconômicas. População Residente. [acessado 2016 Set 4]. Disponível em: http://www2.datasus.gov.br/DATASUS/index.php?area $=0206 \& \mathrm{id}=6942 \& \mathrm{VObj}=\mathrm{http}: / /$ tabnet.datasus.gov.br/cgi/deftohtm.exe?ibge/cnv/pop

12. Instituto Brasileiro de Geografia e Estatística (IBGE). [homepage na internet]. Dados de expectativa de vida. [acessado 2016 Set 4]. Disponível em: http://www. ibge.gov.br/home/

13. Laurenti R, Mello Jorge MHP, Gotlieb SLD. Perfil epidemiológico da morbi-mortalidade masculina. Cien Saude Colet 2005; 10(1):35-46.

14. Neiva KMC, Abreu MM, Ribas TP. Adolescência: facilitando a aceitação do novo esquema corporal e das novas formas de pensamento. PePSIC 2004; 5(2):5664. 
15. Bochner R. Perfil das intoxicações em adolescentes no Brasil no período de 1999 a 2001. Cad Saude Publica 2006; 22(3):587-595.

16. Reichenheim ME, Werneck GL. Anos Potenciais de Vida Perdidos no Rio de Janeiro, 1990. As Mortes Violentas em Questão. Cad Saude Publica 1994; 10(Supl. 1):188-198.

17. Bochner R. Sistema Nacional de Informações TóxicoFarmacológicas - SINITOX e as intoxicações humanas por agrotóxicos no Brasil. Cien Saude Colet 2007; 12(1):73-89.

18. Malaspina FG, ZiniLise ML, Bueno PC. Perfil epidemiológico das intoxicações por agrotóxicos no Brasil, no período de 1995 a 2010. Cad Saude Colet 2011; 19(4):425-434.

19. Soares WL, Freitas EAV, Coutinho JAG. Trabalho rural e saúde: intoxicações por agrotóxicos no município de Teresópolis - RJ. Rev. Econ. Sociol. Rural 2005; 43(4):685-701.

20. Sistema Nacional de Informações Tóxico-Farmacológicas (SINITOX). Dados de Intoxicação. [acessado 2016 Set 4]. Disponível em: http://sinitox.icict.fiocruz.br/dados-nacionais

21. Brasil. Ministério da Saúde (MS). Departamento de Informática. DATASUS. Acesso à Informação. TABNET. Epidemiológicas e Morbidade. Doenças e Agravos de Notificação - 2007 em diante (SINAN). Intoxicação Exógena. [acessado 2016 Set 4]. Disponível em: http:// tabnet.datasus.gov.br/cgi/deftohtm.exe?sinannet/cnv/ Intoxbr.def

22. Associação Brasileira de Saúde Coletiva (Abrasco). Dossiê Abrasco - Um alerta sobre os impactos dos agrotóxicos na saúde. Parte 1 - Agrotóxicos, Segurança Alimentar e Nutricional e Saúde. Rio de Janeiro: Abrasco; 2012

23. Bochner R, Souza VMFA. Panorama das Intoxicações e Envenenamentos Registrados no Brasil pelo Sistema Nacional de Informações Tóxico-Farmacológicas (SINITOX). Revista RACINE 2008; 18(106):44-58.

24. Bombardi LM. Intoxicação e morte por agrotóxicos no Brasil: a nova versão do capitalismo oligopolizado. Bol Dataluta 2011; (45):1-21.

25. Bochner R. Óbito ocupacional por exposição a agrotóxicos utilizado como evento sentinela: quando pouco significa muito. Vigil. Sanit. Debate 2015; 3(4):3949.

26. Bortoletto ME, Bochner, R. Impacto dos medicamentos nas intoxicações humanas no Brasil. Cad Saude Publica 1999; 15(4):859-869.

27. Bernardes SS, Turini CA, Matsuo T. Perfil das tentativas de suicídio por sobredose intencional de medicamentos atendidas por um Centro de Controle de Intoxicações do Paraná, Brasil. Cad Saude Publica 2010; 26(7):1366-1372.
28. Germano LC, Alonzo HGA. Intoxicações e reações adversas a medicamentos: perfil local de subnotificação aos sistemas de informação em saúde. Rev. Elet. Farm. 2015; XII(4):32-44.

29. Vieira DM, Caveião C. Perfil das intoxicações medicamentosas no estado de São Paulo na perspectiva da vigilância sanitária. Rev Saúde Desenvolvimento 2016; 9(5):119-141.

30. Matos GC, Rozenfeld S, Bortoletto ME. Intoxicações medicamentosas em crianças menores de cinco anos. Rev. Bras. Saúde Mater. Infant. 2002; 2(2):167-176.

31. Silber TJ, Souza RP. Uso e abuso de drogas na adolescência: o que se deve saber e o que se pode fazer. Adolesc. Latinoam. 1998; 1:148-162.

32. Minayo MCS, Deslandes SF. A complexidade das relações entre drogas, álcool e violência. Cad Saude Publica 1998; 14(1):35-42.

33. Sanchez ZM, Nappo SA. Seqüência de drogas consumidas por usuários de crack e fatores interferentes. Rev Saude Publica 2002; 36(4):420-430.

34. Heim J, Andrade AG. Efeitos do uso do álcool e das drogas ilícitas no comportamento de adolescentes de risco: uma revisão das publicações científicas entre 1997 e 2007. Rev. Psiquiatr. Clin. 2008; 35(Supl. 1):6164.

35. Ferreira SE, Mello MT, Formigoni MLOS. O efeito das bebidas alcoólicas pode ser afetado pela combinação com bebidas energéticas? Um estudo com usuários. Rev. Assoc. Méd. Bras. 2004; 50(1):48-51.

36. Arraia AM. Increased alcohol consumption, nonmedical prescription drug use, and illicit drug use are associated with energy drink consumption among college students. J Addict Med 2010; 4(2):74-80.

37. Ribeiro DR, Carvalho DS. O padrão de uso de drogas por grupos em diferentes fases de tratamento nos Centros de Atenção Psicossocial Álcool e Drogas (CAPS-AD). J Bras Psiquiatr 2015; 64(3):221-229.

Artigo apresentado em 15/08/2017

Aprovado em 18/06/2018

Versão final apresentada em 20/06/2018 